\title{
Neuroscience and the Brain Institute of Hospital Israelita Albert Einstein
}

\author{
Neurociências e o Instituto do Cérebro do Hospital Israelita Albert Einstein \\ Edson Amaro Junior \\ Guest Editor
}

It is true that we have been noticing a huge progress in the number of publications and, more recently, in the number of citations of scientific papers of neuroscience produced in Brazil. In numbers, we are ranked in $13^{\text {th }}$ place regarding scientific production in neuroscience, and in $18^{\text {th }}$ place as related to the number of citations in this specific field (Scopus www.scimagojr.com / ISI: www.webofknowledge.com). Considering all areas of knowledge, Brazil occupies the $15^{\text {th }}$ place on the list of the biggest publishers of scientific papers worldwide, and the $21^{\text {st }}$ place regarding citations among countries. In Latin America, again, considering only the neuroscience field, our country has approximately 3.2 times more publications and 2.8 times more citations than the one in the second place, Mexico. Similarly regarding production from all areas of knowledge, Brazil has respectively 2.6 and 2.4 times more regarding to publications and citations than the total presented by Mexico. Therefore, neuroscience is one of the areas that is above the national scientific productivity average. Here, the numbers speak for themselves, but, do they say everything? Certainly they do not say how we got there.

There is no doubt that measuring scientific output or "scientometry" is mandatory, and certainly deserves attention and careful consideration.

However, quantitative parameters always involves an unknown error term, and in most cases this term cannot be estimated. Two subjects are not usually quantified, however, maybe should be: the available resources for research funding, and the research cycle - longer than many other productive sectors of society.

I would like to concentrate in some points. Regarding neuroscience in the country (and I believe that this applies to any other area), there are a few relevant factors than the ones listed in the following paragraphs. However, I did choose them due to their representative context.

Brazil has 14 Brain Institutes throughout the country, three of them working with projects in partnership (Universidade Federal de Natal, Pontifícia Universidade Católica do Rio Grande do Sul and Brain Institute (InCe) of Hospital Israelita Albert Einstein, São Paulo). In addition, the São Paulo Research Foundation (FAPESP) holds a program named Inter-institutional Cooperation in Support of Brain Research Program (CInAPCe) that gathers the three main public universities of the state of São Paulo (USP, UNICAMP and UNIFESP) and a non-academic institution, Hospital Israelita Albert Einstein.

Perhaps the most important fact is the increasing phenomenon in neuroscience, 
the scientific production associated to clinical practice. Publications in neuroscience arising from neurology practice have grown at a rate more than double that from in other sources within the neuroscience field.

Considering neuroscience and its subdivisions (measured by international agencies) it is remarkable that basic science grows less than applied science. Articles from the main area of "neurology" are placed in the top of the list of neuroscience publications in Brazil (www.scimagojr.com), without considering the item "miscellaneous" (by the way, a word without a fully known meaning: it may means "a mess" or "something which we do not know the name of", or perhaps, a mix of both...).

It is important to observe that Hospital Israelita Albert Einstein has built along the last eight years the basis for neuroscience research. As a hospital it is natural that the neurologic area be emphasized in its production. Therefore, one can expect that our Hospital is ready to generate leading edge knowledge in neuroscience, particularly in neurology. We are ready for this challenge. But I believe it is necessary to point out some issues for reflection as follows.

The hospital has a research center where the available neuroscience platforms are grouped, and the interchange between basic and applied science has been planned since its foundation. In fact this is one of the InCe's vision. This initiative was unique in our institution when it was created. It is a model of research "verticalization" in a specific knowledge of medical research. The right choices and the wrong ones in the InCe managing process will give directions to similar initiatives in other non-academic institutions also interested in developing research. This seems to be the way followed by other hospitals, both in Brazil and other countries. However, there are challenges, for example, to present to neurologists the possibility to do their research at an institution that has not been academically structured - but instead, chose to follow another pathway. This model is based on meritocracy and in a more representative role for the decisionmaking process.

I believe this type of organization have to rely on collegiate body decisions. It is impossible to make advances without management and specific goals. That is why InCe has a Steering Committee, in which research interests are represented, - and now it is coming at the end of a management cycle. At InCe we already have implemented a structure with leading edge instruments for research, and we have achieved practical results in publications, fundraising, and partnership with international and national institutions for research in neuroscience and further are coming. In this new stage, we look for a higher integration with clinical medicine and also to increase the number of investigators to improve the main subject of research of Instituto Israelita de Ensino e Pesquisa Albert Einstein: studying the ageing process.

Now is the time to bring more physicians to work with clinical research, and to use the resources we have incorporated, not without the efforts of a young and hardworking team.

What does a large hospital earn with this? It generates knowledge in neuroscience. It does not only counts on professionals guided by evidences who apply knowledge, but generates knowledge. At InCe, the platforms of genetic research, nanotechnology, experimental psychopharmacology, functional and structural molecular imaging, brain stimulation and bioinformatics are well established.

In this new phase we must have a more cooperative management to make an even better use the platforms established in neuroscience. We have passed through a phase of implementing structures for basic and applied research, and now, young investigators who have come to the institution are seeking to grow. Attracting and retaining talents is a challenge not only to InCe, but to Sociedade Beneficente Israelita Brasileira Albert Einstein and to the country.

To continue with such initiative requires more risktaking, and right now is the decisive time. We need to grow to follow other leading neuroscience groups in the country, and to contribute for institutional guidelines. In this very moment it is time to invest to retain our young talents. InCe could be the home office for implementing such project as a model.

In summary, organizations need to be ready to benefit from the growing moment experienced by this country 
and by the neuroscience field. InCe represents the key spot to host this growing process at Hospital Israelita Albert Einstein. We need to find new ways to continue to grow. This edition of einstein is an important step forward in this regard, in which the reader will find a few examples of our current research projects. 
